Mathematical and

Computational

PUBLICATIONS

NISTIR 6072

Sciences

Division

\title{
Toward a Revised NBS Handbook of Mathematical Functions
}

Daniel W. Lozier

September 1997

\section{U. S. DEPARTMENT OF COMMERCE \\ Technology Administration \\ National Institute of Standards and Technology \\ Gaithersburg, MD 20899}

$Q C$

100

. $\mathrm{U} 56$

N0.6072 



\section{NISTIR 6072}

\section{Toward a Revised NBS Handbook of Mathematical Functions}

\section{Daniel W. Lozier}

U.S. DEPARTMENT OF COMMERCE

Technology Administration

National Institute of Standards

and Technology

Mathematical and Computational Sciences Division

Information Technology Laboratory

Gaithersburg, MD 20899-0001

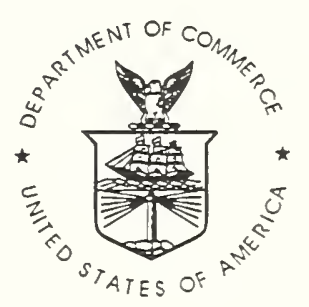

U.S. DEPARTMENT OF COMMERCE

William M. Daley, Secretary

TECHNOLOGY ADMINISTRATION

Gary Bachula, Acting Under Secretary for Technology

NATIONAL INSTITUTE OF STANDARDS

AND TECHNOLOGY

Robert E. Hebner, Acting Director 


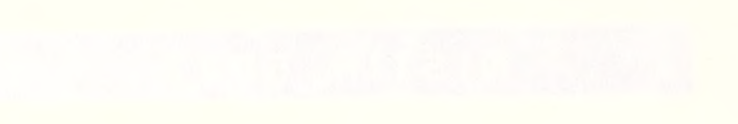

(2) 


\title{
TOWARD A REVISED NBS HANDBOOK OF MATHEMATICAL FUNCTIONS
}

\author{
DANIEL W. LOZIER
}

\begin{abstract}
A modernized and updated revision of Abramowitz and Stegun, Handbook of Mathematical Functions, first published in 1964 by the National Bureau of Standards, is being planned for publication on the World Wide Web. The authoritative status of the original will be preserved by enlisting the aid of qualified mathematicians and scientists. The practical emphasis on formulas, graphs and numerical evaluation will be extended by providing an interactive capability to permit generation of tables and graphs on demand. This report provides background information and early status of the project.
\end{abstract}

\section{INTRODUCTION}

Most would agree that the National Bureau of Standards Handbook of Mathematical Functions has been a phenomenal success in the field of mathematical publishing. Issued in June of 1964 by the US Government Printing Office, this large hardback volume of more than 1000 pages has been in print continuously since that date as a government publication ${ }^{1}$. Over 200 copies were sold in a recent one-year period. A paperback version has been photo-offset and sold by Dover Publications since 1965. Although Dover does not reveal sales data, it undoubtedly outsells the government edition many times over. Both complete and abridged editions (with many of the numerical tables removed) have been produced by other commercial publishers, and translations have appeared in languages other than English (for example, Russian). The handbook is cited very frequently in scientific articles that make use of special functions, making it one of the most cited of all mathematical reference works.

What were the reasons for this success? Four might be comprehensiveness, authoritativeness, timeliness and applicability.

First, comprehensiveness. A concentrated effort was made to identify the most important mathematical functions as measured by their value in fields outside mathematics, such as physics and statistics, and to assemble the most important properties of these functions together with algorithms, tables of numerical values,

Key words and phrases. Handbooks of mathematics, special functions, electronic publication, World Wide Web.

Comments on any aspect of a revised Handbook of Mathematical Functions will be gratefully received. Further information and an e-mail address for providing comments is available at http://math.nist.gov/DigitalMathLib.

This paper was presented at the SIAM Annual Meeting at Stanford University on July 14, 1997, in the Minisymposium on Handbooks for Special Functions and the World Wide Web. The Minisymposium was sponsored by the SIAM Activity Group on Orthogonal Polynomials and Special Functions.

${ }^{1}$ For current information enter the stock number "003-003-00279-8" (with the double-quote characters included) into the search field of http://www.access.gpo.gov/su_docs/sale/prf/prf.html. 
graphical representations and other relevant information. Most of the assembled information had been published previously but it was unified and presented in a consistent format. A serious effort was made to include the latest results from the research literature. In the case of tables of numerical data, accuracy was verified and gaps were filled by new computations.

Authoritativeness was achieved by establishing a supervisory scientific committee with Philip M. Morse of the Massachusetts Institute of Technology as its head. The committee members were A. Erdélyi, M.C. Gray, N.C. Metropolis, J.B. Rosser, H.C. Thacher, Jr., John Todd, C.B. Tompkins and J.W. Tukey. The idea to produce such a reference work originated with Milton Abramowitz of NBS. He developed the overall plan and led the selection of the authors for the various chapters. Irene Stegun, also of NBS, took over as technical editor after the death of Abramowitz. One sign of the authoritative nature of the handbook is the impact it has had on standardizing definitions and notation. All mathematical functions are subject to definitional and notational variations that can be confusing when articles by different authors are compared, especially when different countries or disciplines are involved. By becoming, in effect, the standard reference for special functions in much of the world, the handbook helped reduce this problem.

The appearance of a compendium such as the NBS Handbook was timely in that it appeared at just about the time electronic computers were beginning to make their influence strongly felt in applied mathematics. Thus it served to sum up the state of a highly developed mathematical technology at a time of transition to a new technology. In the earlier technology mathematical problems were considered solved if results could be obtained in terms of elementary or higher functions. Because the properties and behavior of the functions were understood, insight into the nature of the solution was available. This insight could be qualitative or quantitative. Both kinds of insight remain equally valuable today. Qualitative insight comes from viewing the 'forest' at the expense of the 'trees.' A view of the 'forest' coming, for example, from knowledge of the asymptotic behavior or distribution of zeros of functions entering into a solution can be immensely valuable in scientific and engineering applications. On the other hand, in detailed mathematical models of physical processes or engineering structures the 'trees,' e.g. floating-point numbers, are unavoidable. Solutions obtained by more direct methods such as quadrature, finite differences and finite elements provide an immense amount of quantitative information, and their role in scientific computing has ascended with the increasing power of computers since 1964. However, these methods provide no qualitative insight, and competent practitioners still recognize the importance of special functions. Three areas can be listed in which they are important today. First, they provide a source of test problems for direct solvers. Second, they are used to make a computation more efficient in some applications when length and time scales vary over many orders of magnitude through the technique known as subgrid modeling. And third, they can be highly effective when solutions are representable in a series of special functions or as an integral transform.

The final reason for the success of the NBS Handbook is its emphasis on applicability. The functions chosen for inclusion were determined by their importance in applied mathematics, physical sciences, engineering and statistics, both as a vehicle for computation and as a means for intuitive understanding. The approximations 
and numerical methods, including the now obsolete method of interpolation in tables, were oriented toward practical concerns, including the construction and testing of mathematical software. Accordingly, a highly mathematical style with formal definitions, theorems and proofs was avoided while an insistence on mathematical rigor was maintained. Lengthy explanations and mathematical developments were similarly avoided. Thus the style is terse and assumes considerable mathematical capability on the part of the user. The style and choice of content undoubtedly contributed to the broad and continuing appeal of the handbook.

The name we have adopted for the envisioned successor to the NBS Handbook of Mathematical Functions is the NIST Digital Library of Mathematical Functions ${ }^{2}$. Our approach is to retain the ingredients of the original success while taking advantage of a new technological transition. This transition is characterized (in part) by the use of powerful computers and network communications to disseminate highly technical reference information electronically. Electronic publishing, online databases, and generation of technical data on demand are emerging components of this transition. Through its Mathematical and Computational Sciences Division in conjunction with its Electron and Optical Physics Division and its Standard Reference Data Program, NIST can provide the necessary leadership and some of the necessary technical, computational and financial resources. But outside technical and financial assistance is essential, and toward this end an invitational workshop was held July 28-31, 1997, at NIST. The remainder of this report takes a closer look at the existing handbook and examines a few of the issues involved in developing a modernized and updated version.

\section{Existing Scope And Content}

The 29 chapters of the existing handbook are listed in Table 1. Except for a few chapters devoted to introduction, methodology or application, a typical chapter addresses a particular class of special functions.

Five chapters are introductory. Chapters 1 and 2 provide mathematical and physical constants, and conversion factors between metric and US customary units. Chapter 3 covers binomials, progressions, and means; inequalities; formulas from calculus and complex analysis; absolute and relative errors; infinite series; solution of quadratic, cubic and quartic equations; successive approximation methods; and continued fractions. Chapter 4 covers the logarithmic, exponential, circular, inverse circular, hyperbolic and inverse hyperbolic functions. Chapter 28 give basic information on computer arithmetic.

Numerical analysis is the subject of the methodological Chapter 25. It covers forward, central, mean, divided and reciprocal differences; Lagrange, Newton, trigonometric and inverse interpolation, including formulas with throwback and stencils for bivariate interpolation; differentiation formulas, including stencils for partial derivatives and the Laplacian and biharmonic operators; integration formulas, including open and closed Newton-Cotes, Gaussian, and other types of quadrature, and numerous stencils for multidimensional integration; and numerical solution of ordinary differential equations, including Runge-Kutta and predictor-corrector methods. For nearly all formulas in Chapter 25, the corresponding error order, error estimate or error bound is given.

\footnotetext{
${ }^{2}$ In 1988 the National Bureau of Standards became the National Institute of Standards and Technology.
} 


\begin{tabular}{|c|l|}
\hline Chapter & \multicolumn{1}{|c|}{ Title } \\
\hline 1 & Mathematical Constants \\
\hline 2 & Physical Constants and Conversion Factors \\
\hline 3 & Elementary Analytical Methods \\
\hline 4 & Elementary Transcendental Functions \\
\hline 5 & Exponential Integral and Related Functions \\
\hline 6 & Gamma Function and Related Functions \\
\hline 7 & Error Function and Fresnel Integrals \\
\hline 8 & Legendre Functions \\
\hline 9 & Bessel Functions of Integer Order \\
\hline 10 & Bessel Functions of Fractional Order \\
\hline 11 & Integrals of Bessel Functions \\
\hline 12 & Struve Functions and Related Functions \\
\hline 13 & Confluent Hypergeometric Functions \\
\hline 14 & Coulomb Wave Functions \\
\hline 15 & Hypergeometric Functions \\
\hline 16 & Jacobian Elliptic Functions and Theta Functions \\
\hline 17 & Elliptic Integrals \\
\hline 18 & Weierstrass Elliptic and Related Functions \\
\hline 19 & Parabolic Cylinder Functions \\
\hline 20 & Mathieu Functions \\
\hline 21 & Spheroidal Wave Functions \\
\hline 22 & Orthogonal Polynomials \\
\hline 23 & Bernoulli and Euler Polynomials, Riemann Zeta Function \\
\hline 24 & Combinatorial Analysis \\
\hline 25 & Numerical Interpolation, Differentiation and Integration \\
\hline 26 & Probability Functions \\
\hline 27 & Miscellaneous Functions \\
\hline 28 & Scales of Notation \\
\hline & Laplace Transforms \\
\hline
\end{tabular}

TABLE 1. Chapters of the NBS Handbook of Mathematical Functions.

Three chapters are oriented toward applications. Chapter 24 addresses combinatorial analysis (binomial and multinomial coefficients; partitions of integers; and Mobius and Euler functions). Chapter 26 deals with statistics (definitions and properties of distribution functions; normal and bivariate normal probability functions; and chi-square, incomplete beta, variance-ratio, and Student's $t$-distributions). Finally, chapter 29 concerns Laplace transforms (definitions and formulas; and short tables of Laplace and Laplace-Stieltjes transforms). 
The remaining 20 chapters form the core material of the handbook. Each treats an individual class of special functions, and typically each is divided into four sections. The first section, Mathematical Properties, presents definitions, differential equations, integral representations, recurrence relations, functional relations, series expansions, continued fractions, asymptotic expansions, polynomial approximations, special values, derivative formulas, integrals, zeros, graphical representations, and so on. The second section, Numerical Methods, gives advice on how to compute function values effectively by using numerical tables, interpolation methods, and relevant mathematical properties such as recurrence relations. The third section, References, is divided into two parts, one for texts and the other for tables. The final section, Tables, contains data taken from previously published sources, verified and augmented with computations performed at NBS.

\section{Opportunities and OBStaCles}

In the introduction to this report an allusion was made to an unfolding revolution in information technology which is marking a new technological transition. This revolution is being driven by technological advances in hardware, software, and especially in digital communications. This report is not the place to try to define or describe this revolution in general terms. Everyone is well aware of its existence and of its potential for dramatic impacts in book publishing, journal publishing, interpersonal communication, and dissemination of all kinds of information. Indeed, the impact has already been enormous. In mathematical publishing, LATEX ${ }^{3}$ is used routinely to typeset arbitrarily complex material. Diskettes and compact disks (CD-ROMs) are distributed with published books to provide relevant computer files to the purchaser. Automated repositories are used to obtain software documents and other material by a simple electronic request; the requester is the only human in the transaction. These are examples of one-way communication from an author to a reader or 'information consumer' in which new technology results in an improved product. The information transferred (mathematical text, graphs, tables of numbers, programs) is prepared in advance in the expectation that it will meet future needs, and of course there are always unanticipated needs that are not met.

What is needed to meet unanticipated needs is a mechanism that can generate and deliver information on demand. This is achievable using the World Wide Web (WWW) in conjunction with computer programs. In a typical transaction, the consumer enters a request for information on a 'question page,' and an 'information provider' returns an 'answer page.' The answer page could be a simple image of a record extracted from a database (reminiscent of one-way communication between an author and a reader), or it could be generated by an arbitrarily complicated computation. To get beyond the traditional concept of a fixed and immutable publication, the term digital library $y^{4}$ has come into use.

There are opportunities and obstacles in applying digital library technology to the subject matter of the NBS Handbook. The overall opportunity is to provide to the widest possible audience an authoritative and up-to-date resource center for special functions. We envision constructing a resource center, or digital library, in two major stages. The first stage will be similar in concept to the existing handbook with as many extensions as possible in the direction of dynamic generation of

\footnotetext{
${ }^{3}$ L. Lamport, LATEX Document Preparation System, Second Ed., Addison-Wesley, 1994

${ }^{4}$ For example, see http://www.nsf.gov/od/lpa/news/publicat/frontier/5-97/5digital.htm.
} 
information. A large database of static answer pages will be constructed and used as the basis for satisfying information requests. The same database could serve as the basis for books and CD-ROMs. An immediate advantage of CD-ROMs and the WWW over books is the possibility of downloading crucial artifacts, such as symbolic code for mathematical formulas, into documents and computer programs. In this initial stage a major obstacle will be in connection with the numerical tables. First, the precision and range of the existing tables are inadequate to current needs, particularly when used to validate numerical software. Second, software packages exist that can compute vastly extended numerical tables-but the state of the art in software packages today is such that reliability of the computed values remains open to question. The decision of what to include in the way of numerical tables, if any, will be difficult. References without endorsement to algorithms and software can be given and will be useful but a completely satisfactory outcome with respect to the numerical tables is not likely in the initial stage.

In the second stage we envision making a serious attempt to overcome the numerical tables obstacle. The idea is to develop a standard reference library of numerical software that is capable of computing function values to at least quadruple precision over very large ranges of the input arguments and parameters. But we require even more - that the accuracy of quadruple precision is reliable in the sense that all errors arising from truncation and rounding are monitored and kept under strict control. This requirement is necessary to uphold the standard of authoritativeness that is desirable for a genuine successor to the NBS Handbook. The library will be used to generate numerical tables on demand.

The obstacles to the construction of such a library are mathematical and computational. Algorithms with strict bounds on truncation and rounding errors are not generally available for special functions. The techniques of interval analysis are applicable here but most of the effort in that field has been in other directions. The flaws in computer arithmetic systems, such as their vulnerability to underflow and overflow, make it difficult to construct robust and reliable software. But these obstacles provide an opportunity for creative mathematicians and computer scientists to contribute toward the solution of an important problem: replacing standard tables with standard software of comparable quality.

The use of standard tables to validate computer software was mentioned earlier. A large effort to develop advanced test procedures for mathematical software is underway in the Information Technology Laboratory at NIST. One component of this effort is a Software Test Service for Special Functions ${ }^{5}$. This service will use the WWW to help software users and developers craft detailed tests based on comparison to a standard. The most important missing piece is a standard reference library for special functions. Stopgap measures are being used until such software becomes available. As the second stage of our anticipated project progresses, the test service is expected to become an integral part of the NIST Digital Library of Mathematical Functions.

Graphics, applications of special functions, and the role of symbolic processing are important also. For graphics, as with tables, dynamic generation of information promises useful benefits. For example, fixed graphs could be replaced by graphical objects that could be manipulated (rotated, enlarged, viewed in cross sections, etc.)

\footnotetext{
${ }^{5}$ For further information see http://math.nist.gov/stssf.
} 
under the control of commands given by the user. In the area of applications, an exciting opportunity exists to improve communication between mathematicians and scientists. The notation and normalization conventions used in different fields can lead to irritating difficulties in understanding as well as actual errors. We envision a set of application digital libraries that call upon the Digital Library of Mathematical Functions for standard data. These data will be translated into a form that is immediately meaningful to scientists in the application area. Toward this end, and as a prototype for future developments, our project plan includes construction of application digital libraries of electromagnetism and quantum physics by the Electron and Optical Physics Division of NIST. Finally, symbolic processing is expected to be useful also in helping users resolve differences in notation and normalization, and for other purposes. User controls, as in the case of manipulating graphs, are applicable here as well.

\section{Prospectus}

A reference work like the NBS Handbook can be made even more valuable by putting it on the WWW. This theme has been explored, with attention paid to obstacles as well as opportunities.

The basic content of the handbook is found in the Mathematical Properties sections of the core chapters. A well-funded project to revise the handbook will provide the opportunity to update these sections to take account of mathematical developments of the last 30-plus years. For example, the chapter on elliptic integrals predates the influential work of Carlson that not only supplanted the standard functions of Legendre with more satisfactory symmetric functions but also yielded effective algorithms that are now found in virtually all computer libraries of special functions. And there have been important developments in discrete orthogonal polynomials, basic and generalized hypergeometric functions, and q-functions that are not covered at all in the existing handbook. We plan to follow the proven success of Abramowitz's approach: contract with key mathematicians who can do a first-rate job of writing the chapters of the new handbook, under the supervision of a carefully selected panel of senior research mathematicians and scientists who have a proven record of accomplishment with respect to special functions. We plan to add to this approach a very strong validation process, supervised by the same panel, in which the chapters are peer-reviewed independently of the authors. Independent validation was not a feature of the original handbook project but was used, for example, with great success in the later development of the NAG Library ${ }^{6}$. Additional informal reviews and comments from the mathematical and scientific community at large will provide still further validation.

We envision a concentrated effort over a 2-3 year period, after which the initial stage of the handbook revision project will be complete. This period will begin upon receipt of adequate funding authority. The initial stage will cover all the existing handbook, with extensions, but not with standard reference software substituted for standard reference tables. The second stage is in some ways already started through its connection to the Software Test Service for Special Functions. However, much research and development is needed before an acceptable standard library for the

\footnotetext{
${ }^{6}$ B. Ford and J.C.T. Pool, The Evolving NAG Library Service, in W.R. Cowell (Ed.), Sources and Development of Mathematical Software, Prentice-Hall, 1984, pp. 375-397.
} 
numerical generation of special functions can be constructed. Therefore the second stage will undoubtedly continue well beyond the completion of the initial stage.

\section{ACKNOWLEDGMENTS}

The author wishes to acknowledge the eleven other members of the NIST Committee that has been meeting informally to consider the possibility of revising the NBS Handbook: Paul Boggs, Ron Boisvert, Charles Clark, Judy Devaney, Leonard Maximon, Bruce Miller, Peter Mohr, Frank Olver, John Rumble, Barry Schneider and Charles Sturrock.

The author wishes also to acknowledge Marjorie McClain and Bonita Saunders of NIST, and Yuri Rappoport of the Russian Academy of Sciences. They are active contributors to the project that is developing the Software Test Service for Special Functions, as are several of the people acknowledged above.

Further acknowledgment is due Richard Askey, Ross Barnett, Claude Brezinski, Bill Carlson, Walter Gautschi, Joe Jenkins, Kurt Kölbig, Ingram Olkin and Nico Temme. These are the non-NIST invitees to the Invitational Workshop on the NIST Digital Library of Mathematical Functions, which was held July 28-31, 1997, at NIST in Gaithersburg, MD.

Finally, acknowledgment is due to the many people from around the world who have communicated with the author in different ways.

Mathematical Software Group, Mathematical and Computational Sciences Division,

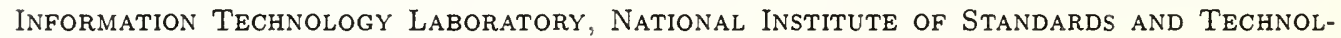
OGY, Gaithersburg, MD 20899

E-mail address: dlozier@nist.gov 

